\title{
Un esquema conceptual para identificar localidades com poblaciones em riesgo de anemia y desnutrición crónica
}

\author{
Andrea Liliana Vesga Varela ${ }^{1}$, Helena Pachón ${ }^{2}$, Glenn Hyman ${ }^{3}$, \\ Fredy Alexander Monserrate Rojas ${ }^{4}$, Emmanuel Zapata-Caldas ${ }^{5}$
}

Datos nacionales e incluso departamentales de anemia y desnutrición crónica se recolectan con periodicidad. Es raro encontrar datos a nivel municipal del estado nutricional de una población, información necesaria para focalizar intervenciones. Un esquema conceptual se desarrolló, aplicó y válido. Datos bioquímicos (prevalencia departamental de hemoglobina infantil $<11 \mathrm{~g} / \mathrm{dL}$ ), antropométricos (prevalencia departamental de talla/edad infantil $<-2$ Desviación Estándar) y socioeconómicos (a nivel municipal, índice de intensidad de pobreza ó población bajo la línea de pobreza extrema) se usaron para identificar localidades con riesgo de presentar anemia y desnutrición crónica, en 11 países latinoamericanos. En un sistema de información geográfica, se unificaron datos nutricionales y socioeconómicos a un mismo formato espacial, que representaba una localidad en un determinado departamento de un país. Se ubicaron aquellas localidades donde coincidían alta desnutrición (anemia o crónica) y pobreza. Para la desnutrición crónica, hubo una alta relación de localidades identificadas con el esquema, al compararlas con datos recolectados a nivel municipal $(\geq 66 \%)$, mas no cuando se comparó con un método estadístico $(0 \%)$. Este esquema articulado a un software de mapeo facilitó la identificación de localidades con poblaciones en riesgo a anemia y desnutrición crónica. Es importante validar el esquema con estudios de campo.

Palabras-clave: indicadores nutricionales, riesgo nutricional, diagnóstico poblacional, hemoglobina, baja talla.

\section{A conceptual framework for identifying localities at risk for anemia and stunting}

National and regional-level data on anemia and stunting are regularly collected. Local-level data on a population's nutritional status are rarely available. For targeting, methods are needed to identify localities with populations at risk for undernutrition. A conceptual framework was developed that used biochemical (departmental prevalence of child hemoglobin $<11 \mathrm{~g} / \mathrm{dL}$ ), anthropometric (departmental prevalence of child height/age $<-2$ Standard Deviation), or socioeconomic data (municipal poverty intensity index or, when unavailable, the prevalence of extreme poverty) to identify localities at risk for anemia and stunting in 11 Latin American and Caribbean countries. In a geographic information system, all data were adjusted to the same spatial format, a $100 \mathrm{~km}^{2}$ pixel which represents a locality in a particular country's department. The resulting maps highlight those localities with a high prevalence of malnutrition (anemia or stunting) and poverty. In validating the framework with municipal-level data from two Mexican states, $\geq$ $66 \%$ of the localities coincided. Comparing the framework with municipal-level statistical estimations resulted in $0 \%$ coincidence. This framework coupled with mapping software facilitates the identification of localities with at-risk populations for anemia and stunting. The framework should be validated with field studies.

Key-words: nutrition indicators, nutritional risk, population assessment, hemoglobin, stunting.

\footnotetext{
${ }^{1}$ Licenciada em Nutrición y Dietetica, Universidad Industrial de Santander (UIS), Bucaramanga, Colombia.

${ }^{2}$ PhD em Nutrición y MPH, Centro Internacional de Agricultura Tropical (CIAT), Cali, Colombia. Helena Pachón, CIAT, AA 6713, Cali, Colombia. Teléfono + 572 445 0000, Fax + 572445 0073. Email: h.pachon@cgiar.org.

${ }^{3}$ PhD em Geografía, Centro Internacional de Agricultura Tropical (CIAT), Cali, Colombia.

${ }^{4}$ Ingeniero Agrónomo, Centro Internacional de Agricultura Tropical (CIAT), Cali, Colombia.

${ }^{5}$ Licenciado em Geografía, Centro Internacional de Agricultura Tropical (CIAT), Cali, Colombia. Universidad del Valle, Cali, Colombia.
} 


\section{INTRODUCCIÓN}

Pese a las mejorías nutricionales en América Latina y el Caribe en las últimas décadas, aún existen problemas nutricionales en la región [1]. Dos de los mayores problemas de desnutrición en Latinoamérica y el Caribe son la anemia y desnutrición crónica (baja talla para la edad) [2]. En varios países de la región y para diferentes grupos etarios, la prevalencia de anemia supera el $40 \%$ (considerado un problema severo de salud pública en diferentes países): Belice (embarazadas), Bolivia (preescolares), Brasil (preescolares), Haití (preescolares, embarazadas, mujeres en edad fértil), Panamá (mujeres en edad fértil) y Perú (preescolares, embarazadas, mujeres en edad fértil) [3]. A su vez, los países latinoamericanos y caribeños con la mayor prevalencia de desnutrición crónica son Perú (21.7\%), Haití (21.9\%), Bolivia $(24.1 \%)$ y Guatemala $(41.9 \%)$ [4]. La anemia, en particular aquella provocada por una deficiencia de hierro, afecta el desarrollo motor en niños, reduce la capacidad física en adultos y aumenta la morbilidad y mortalidad materna en embarazadas [5]. Las consecuencias de la desnutrición crónica también abarcan múltiples grupos etarios: menor empeño cognitivo y académico en niños, menor productividad económica en adultos, y mayor mortalidad en infantes nacidos de mujeres con baja estatura ${ }^{6}$.

Intervenciones para abordar estos problemas, tendrán mayor éxito en aquellos lugares donde el riesgo es alto a padecer de los mismos. Las encuestas de demografía y salud [4] proporcionan información nacional y sub-nacional de la prevalencia de desnutrición, igual que encuestas nacionales de nutrición [7,8]. Estas usan criterios y puntos de corte internacionalmente reconocidos [9,10] para facilitar la comparación de desnutrición entre países, y de año en año.

Dentro de políticas de seguridad alimentaria del gobierno estadounidense para Latinoamérica y el Caribe, se recomienda "el fortalecimiento de programas nutricionales comunitarios", enfocado especialmente en la reducción de la desnutrición crónica [11]. Para que estos programas comunitarios tengan éxito, se tienen que identificar aquellas comunidades donde la desnutrición es un problema importante [12]. No obstante, para aquellos programas o proyectos que abarquen grandes extensiones geográficas (i.e., departamentos), no siempre es posible realizar un levantamiento de datos que determine el estado nutricional de la población objetiva. En estos casos, es útil poder usar datos existentes que identifiquen aquellas localidades con mayor riesgo a la anemia y a la desnutrición crónica. Con pocas excepciones en la región latinoamericana y caribeña ${ }^{[13]}$, la prevalencia de anemia y desnutrición crónica sólo se conoce a nivel departamental o nacional [14].

El proyecto AgroSalud (www.AgroSalud.org) pretende disminuir en varios países latinoamericanos y caribeños la prevalencia de anemia y desnutrición crónica, entre otros, a través de cultivos mejorados nutricionalmente [15]. Los cultivos se mejoran a través de un proceso natural de cruzamiento de variedades en campos agrícolas [16]. Diversas investigaciones con cultivos biofortificados demuestran el impacto potencial o actual de estos cultivos en mejorar estado nutricional. Por ejemplo, modelajes matemáticos con datos dietéticos de México [17], Colombia [15,16,18] y Bangladesh [19] indican que cultivos biofortificados con hierro y zinc podrían aumentar significativamente el consumo de estos nutrientes en la población. Mujeres filipinas no-anémicas que consumieron diariamente por nueve meses arroz biofortificado con hierro tuvieron un $20 \%$ de aumento en el hierro almacenado (ferritina) en su cuerpo [20]. Escolares mexicanos presentaron una mejoría en el receptor de transferritina al consumir frijol biofortificado con hierro por seis meses [21]. En mujeres de edad fértil en México, el trigo biofortificado con zinc incrementó en 33\% el zinc absorbido por las mujeres [22]. En un meta-análisis de ocho estudios realizados en América Latina y África se encontró que el maíz biofortificado con lisina y triptófano mejoró en un $9 \%$ la talla de preescolares con desnutrición leve o moderada [23]. Las personas que más se beneficiarán de esta intervención son aquellas que padecen de desnutrición en zonas agrícolas, donde se puedan sembrar los cultivos mejorados. Por eso, surgió la necesidad de determinar al nivel geográfico más pequeño y utilizando datos existentes, aquellos lugares donde la prevalencia de anemia y desnutrición crónica fueran elevadas.

El objetivo de esta investigación fue establecer y evaluar la aplicabilidad de un esquema conceptual proyectado para clasificar a nivel local el riesgo poblacional de anemia y desnutrición crónica, utilizando datos secundarios en 11 países latinoamericanos y caribeños.

\section{MÉTODOS}

Datos. Se adquirieron bases de datos e informes de encuestas de demografía y salud, de nutrición y censos 
de talla de 11 países latinoamericanos y caribeños (Tabla 1) que forman parte del consorcio del proyecto AgroSalud. La mayoría de los datos antropométricos se encontraban analizados con las curvas anteriores de la Organización Mundial de la Salud (OMS) [24]. Para la clasificación de los datos antropométricos, según los índices e indicadores propuestos abajo, se aplicaron las curvas actuales de crecimiento y el software de la OMS
[25] para analizar las mismas. La excepción fue México [13] y República Dominicana [26] en cuyos datos ya se habían aplicado. El índice bioquímico creado fue hemoglobina $<11 \mathrm{~g} / \mathrm{dL}$ [9] y el antropométrico fue bajo $(<-2$ Desviación Estándar - DE) talla/edad [10]. Con una excepción [13], los datos bioquímicos y antropométricos sólo tenían representación geográfica a nivel regional, departamental, provincial o estatal.

Tabla 1. Fuentes de información para aplicar el esquema a los 11 países latinoamericanos e caribeños del estudio.

\begin{tabular}{|c|c|c|c|}
\hline \multirow{2}{*}{ País } & \multicolumn{3}{|c|}{ Fuentes de datos } \\
\hline & Hemoglobina & Antropométricos & Socioeconómicos* \\
\hline Bolivia & [35] & [35] & [27] \\
\hline Brasil & Sin dato & [42] & [27] \\
\hline Colombia & [7] & [7] & [27] \\
\hline El Salvador & [36] & [36] & {$[27]$} \\
\hline Guatemala & [37] & [37] & [27] \\
\hline Haití & [38] & [38] & Sin dato \\
\hline Honduras & [39] & [39] & [27] \\
\hline México & {$[8]$} & [13] & {$[27]$} \\
\hline Nicaragua & [40] & [40] & [27] \\
\hline Perú & [41] & [41] & [27] \\
\hline República Dominicana & Sin dato & [26] & [29] \\
\hline
\end{tabular}

\footnotetext{
* Los datos socioeconômicos se refieren al índice de pobreza ${ }^{[27]}$ y al porcentaje de la población bajo la línea de pobreza extrema ${ }^{[29]}$.
}

Los datos socioeconómicos provenían principalmente de censos nacionales de población y vivienda, y de encuestas para la medición del nivel de vida, organizados en una base de datos. Éstos correspondían al índice de intensidad de pobreza y al porcentaje de la población bajo la línea de pobreza extrema. El primero es un índice que consiste en un promedio ponderado del porcentaje de habitantes en hogares con 1, 2, 3 ó 4 Necesidades Básicas Insatisfechas (NBI), donde hogares con un mayor número de NBI tiene un índice más alto [27]. El índice de NBI contiene información sobre acceso a vivienda, a servicios sanitarios, a educación y capacidad económica [28]. Estos datos se tomaron de un estudio realizado por Schuschny \& Gallopín para Meso América y Sur América [27]; por tanto son los mismos índices en todos los países. El porcentaje de la población bajo la línea de pobreza extrema se define como la proporción de la población cuyos ingresos (o consumo) son menores a USD1,25 diario [29]. Todos los datos socioeconómicos encontrados tenían representación geográfica municipal.

Terminología y esquema conceptual. Se utilizaron los siguientes términos según la Food and Agriculture Organization (FAO) [30]: Mediciones brutas (e.g., hemoglobina, talla) e índices calculados (e.g., hemoglobina $<11 \mathrm{~g} / \mathrm{dL}{ }^{[0]}$, talla/edad $<-2$ DE ${ }^{[10]}$. A partir de ellos se establecieron indicadores de riesgo poblacional (e.g., prevalencia de anemia, prevalencia de desnutrición crónica) y categorías de riesgo poblacional según prevalencia: bajo $(<20 \%)$, moderado $(20-39 \%)$ y alto $(\geq 40 \%)$ para anemia ${ }^{[31]}$ y baja $(<10 \%)$, media $(10-$ 
$19 \%)$, elevada (20-29\% y muy elevada $(\geq 30 \%)$ para desnutrición crónica [30].

Se estructuró un esquema conceptual que permitiera llenar con datos fácilmente disponibles, sin tener que recurrir a la recolección de datos primarios, la clasificación de localidades dentro de países, de acuerdo al riesgo (i.e., bajo, moderado, elevado) a sufrir anemia y desnutrición crónica. En el esquema se evalúan simultáneamente dos tipos de información [32]: nutricional y socioeconómico. En el caso de anemia, se agrupan datos de la prevalencia de anemia con los de la situación socioeconómica (índice de pobreza o el porcentaje de la población por debajo de la línea de pobreza extrema). Estos se reúnen a un nivel geográfico de $100 \mathrm{~km}^{2}$ y en un sistema de información geográfica, como se describe posteriormente, para identificar aquellas localidades donde coincide la desnutrición elevada con la pobreza elevada. En la práctica lo que esto implica es que, tanto la prevalencia departamental de anemia como la prevalencia de bajo nivel socioeconómico del municipio, se aplica a todas las localidades que se encuentran en pixeles de $100 \mathrm{~km}^{2}$. Para identificar localidades con alto riesgo a sufrir desnutrición crónica, se siguió el mismo procedimiento, agrupando la prevalencia de desnutrición crónica con la de bajo nivel socioeconómico en espacios geográficos de $100 \mathrm{~km}^{2}$.

Mapeo de los resultados. Para realizar el proceso de superposición ponderada o weighted overlay de ArcGIS 9.2 ${ }^{\circledR}$ (Redlands, Estados Unidos) de las variables nutricionales y socioeconómicas empleadas en este trabajo, se necesitó que éstas estuviesen un formato espacial denominado ráster, que son celdas o píxeles de igual tamaño que componen una grilla. Los datos existentes (prevalencia departamental de hemoglobina infantil < $11 \mathrm{~g} / \mathrm{dL}$ y prevalencia departamental de talla/edad infantil <-2 DE, índice de pobreza y prevalencia de pobreza extrema) que se encontraban en formato vectorial, referidos a polígonos, puntos y líneas, pero que para este caso sólo comprendía polígonos, se convirtieron al formato ráster. Como resultado se obtuvo un nuevo archivo, o resultado del proceso, que representó las localidades con poblaciones en riesgo de anemia y desnutrición crónica con una resolución de píxel de $100 \mathrm{~km}^{2}$.
Al trabajar con diferentes tipos de variables (i.e., por un lado, prevalencias nutricionales de hemoglobina y talla/edad, y por el otro, índices de intensidad de pobreza y población bajo la línea de pobreza extrema) fue necesario reclasificar los valores de cada una para obtener un rango común y realizar el proceso de superposición ponderada. En otras palabras, se normalizaron los valores de las variables en una escala de 3 a 9 (i.e., los píxeles tomaron valores dentro de este rango), donde 3 fue bajo, 6 moderado y 9 alto ${ }^{[31]}$ en el caso de la prevalencia de anemia infantil y de los datos socieconómicos. En la reclasificación para la prevalencia de desnutrición crónica infantil, 3 fue bajo, 5 moderado, 7 alto y 9 muy alto [30].

En el siguiente paso se asignaron pesos de influencia a cada variable. No obstante, en este caso, se determinó el 50\% a cada una al considerar ambas de igual importancia para la selección de localidades con poblaciones en riesgo de anemia y desnutrición crónica. El mapa resultante del proceso de superposición ponderada arrojó valores entre 3 y 9 a cada píxel. Los resultados se perfeccionaron con la aplicación de un filtro que eliminaba los píxeles aislados y agrupaba los que se hallaban cerca. Finalmente, los píxeles con valores más altos ( 9 , o en su defecto 8 ó 7 , y así sucesivamente, dependiendo del valor más alto de mapa resultado) se escogieron para configurar el mapa final de poblaciones en riesgo de anemia y desnutrición crónica.

Un ejemplo del proceso seguido es el siguiente (Figura 1): La prevalencia de anemia e intensidad de pobreza, se reclasificaron en una escala común de números enteros, entre 3 y 9. Posteriormente, se multiplicó el valor de cada píxel por la ponderación de cada variable (0.50). Asumiendo que el valor de cada píxel es 9, en ambas variables se multiplicó lo siguiente: $9 * 0.50=4.5$ (anemia) y $9 * 0.50=4.5$ (pobreza). Luego los resultados se sumaron, dando 9, el valor del píxel de salida. Sin embargo, en este ejemplo, se ilustra únicamente el caso de un píxel en cada una de las variables en cuestión, más no el resultado final que es la superposición de todos los píxeles en ambas variables. Debido a que el resultado generó un nuevo mapa con valores entre 3 y 9, se tomó la categoría con el valor más alto para definir las localidades con poblaciones en riesgo de anemia. 


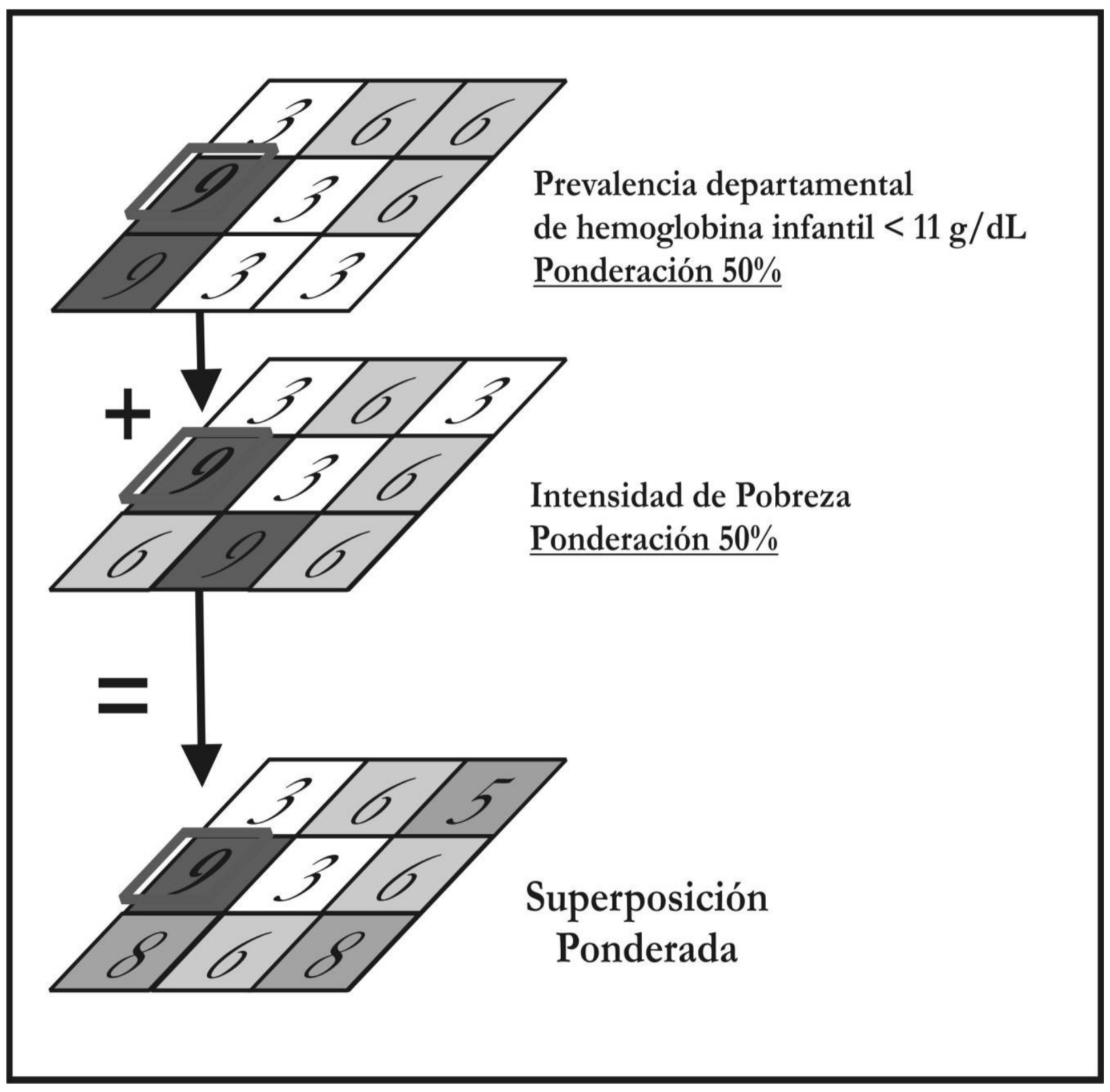

* En este proceso, se multiplicó el valor de cada píxel por el porcentaje de influencia de la variable (i.e., 50\% para ambas). En la figura, cada lámina hace referencia a la distribución de la variable en un país determinado, y el píxel ubicado de izquierda a derecha en la convergencia de la segunda línea y la primera columna, de las variables prevalencia departamental de hemoglobina infantil $<11 \mathrm{~g} / \mathrm{dL}$ e intensidad de pobreza, se multiplicó por su respectivo porcentaje de influencia. Como resultado, se obtuvo un nuevo valor para el píxel resultante en la misma posición del mapa de superposición ponderada. El píxel con valor más alto (en este caso 9), se tomó para configurar el mapa final de localidades con poblaciones en riesgo de anemia. Este mismo proceso se llevó a cabo reemplazando la prevalencia departamental de talla/edad infantil por prevalencia departamental de hemoglobina infantil para obtener un mapa de localidades, en cada uno de los países involucrados en el análisis, con poblaciones en riesgo de desnutrición crónica.

Figura 1. Proceso de superposición ponderada de variables*.

Evaluación del esquema. Se evaluó el esquema de dos maneras: contra un estándar de oro (prevalencia conocida de desnutrición a nivel municipal) y contra un método estadístico indirecto. Primero, con datos 
municipales de la talla de escolares en México (Figura 2D) [13] se estimó la prevalencia de desnutrición crónica en cada estado del país (Figura 2A). Luego, se aplicó el esquema a estos datos seleccionando aquellos estados con mayor prevalencia de desnutrición crónica y cruzando esa información con datos socioeconómicos de intensidad de pobreza (Figura 2B, información disponible a nivel municipal) para identificar los municipios con mayor riesgo a desnutrición crónica (Figura 2C). Estos municipios se compararon con aquellos de mayor prevalencia de desnutrición crónica, según el censo nacional de talla [13].

La segunda evaluación se hizo con estimaciones realizadas por Rogers et al. [33] de los municipios con mayor desnutrición crónica en la República Dominicana. Con datos antropométricos y del censo nacional, ambos recolectados en el 2002, Rogers y colegas crearon un mapa, entre varios, con la prevalencia municipal de baja talla/edad, cruzada con la desigualdad económica. El primero se estimó usando la técnica estadística denominada Estimaciones de Áreas Pequeñas (EAP); las proyecciones de la prevalencia municipal de desnutrición se basaron en modelos de regresión de diferentes variables encontradas en el censo, y en la encuesta demográfica y de salud. Se compararon los municipios que surgieron de estos investigadores con aquellos identificados a través de la aplicación del esquema conceptual (Figura $3 \mathrm{E})$.

\section{RESULTADOS Y DISCUSIÓN}

Esquema conceptual. En las Figuras 2C, 3 y 4 se observa la aplicación del esquema conceptual. Con el esquema, se identificaron municipios con riesgo a desnutrición crónica (Figura 2C) por la asociación de datos nutricionales a nivel departamental (Figura 2A) con datos socioeconómicos a nivel municipal (Figura 2B). En Colombia hubo varios municipios en el departamento de Córdoba con riesgo a desnutrición crónica (Figura 3A). En el caso del Perú, los municipios con mayor riesgo se encontraron principalmente en el nororiente, en los departamentos de Loreto y Amazonas, además de algunos municipios en la zona andina de Cuzco, Apurimac y Huanuco (Figura 3B). Con riesgo a desnutrición crónica se encontraron varios municipios en el norte brasileño (Figura 3C), con una concentración alta en esta región y una dispersión de municipios en el Nordeste. El Salvador tuvo el mayor número de municipios con riesgo en los departamentos de Ahuchapán y Cuscatlán (Figura 3D). En la República Dominicana, una franja de municipios en la provincia Elias Pina, fronteriza con Haití, y en la provincia Baoruco que colinda con ésta, resultan con mayor riesgo nutricional (Figura 3E).

Agrupando datos de hemoglobina y socioeconomía, se identificaron municipios con riesgo a la anemia (Figura 4). En Colombia, éstos se encuentran en varios departamentos de la Costa Atlántica (Figura 4A). En Perú, municipios de departamentos en el norte (Amazonas y Piura, principalmente) y la Amazonía central (Ucayali y Madre de Dios en particular) del país tienen riesgo a la anemia (Figura 4B). En México, apareció un gran número de municipios con riesgo a la anemia, concentrados en el centro y sur, pero también surgieron algunos en el norte del país (Figura 4C). En El Salvador hubo una mayor dispersión de municipios con riesgo a la anemia, incluyendo los departamentos de La Unión, Chalatenango y Ahuchapán (Figura 4D).

Validación del esquema. Aplicando el esquema conceptual, se identificaron 97 municipios en el estado de Chiapas, México con riesgo a desnutrición crónica (Figura 2C). Realizando una comparación con el estándar de oro, se observó que la medición directa de la estatura de los niños identificó 39 municipios en Chiapas con más de un 40\% (riesgo muy elevado) de prevalencia de baja talla/edad (Figura 2D). De estos 39 municipios, el esquema conceptual identificó 34 (87\%) con riesgo a la desnutrición crónica (Figura 2C). Por su parte, para el Estado de Oaxaca, el análisis se hizo a nivel de distrito (en México, unidad administrativa compuesta por municipios). De los 33 distritos que hacen parte de Oaxaca, se encontraron 29 con más del $40 \%$ de los niños con baja talla/edad (Figura 2D) de acuerdo con la medición directa. Los resultados de la aplicación del esquema identificó 21 distritos con niños en riesgo a desnutrición crónica (Figura 2C), de los cuales $19(66 \%)$ coinciden con los 29 distritos identificados con medición directa. El esquema identificó más lugares en riesgo de desnutrición crónica que la medición directa en Chiapas y lo opuesto en Oaxaca.

Comparando el esquema final con la metodología indirecta EAP en la República Dominicana, el municipio donde coinciden los cuartiles con mayor desnutrición y desigualdad, se encuentra en la provincia Elias Pina [33]; es decir, similar 
a la Figura 3E. Sin embargo, el uso de EAP encontró que el municipio con mayor desnutrición según baja talla/edad (prevalencia entre 40 y $60 \%$ ) se encuentra en la provincia de Hato Mayor, seguido por municipios en varias provincias del país, con una prevalencia de baja talla/edad entre 20 y $40 \%$. La mayoría de éstos se encuentran en la zona occidental del país. Es decir, la vinculación de datos antropométricos a nivel provincial con datos de pobreza a nivel municipal (Figura 3E) no arrojó los mismos municipios en riesgo nutricional según la técnica EAP. Resultados de técnicas como la EAP pueden ser sesgados debido a la muestra que contribuye datos al modelo, y los supuestos del modelo estadístico aplicado [34].

A

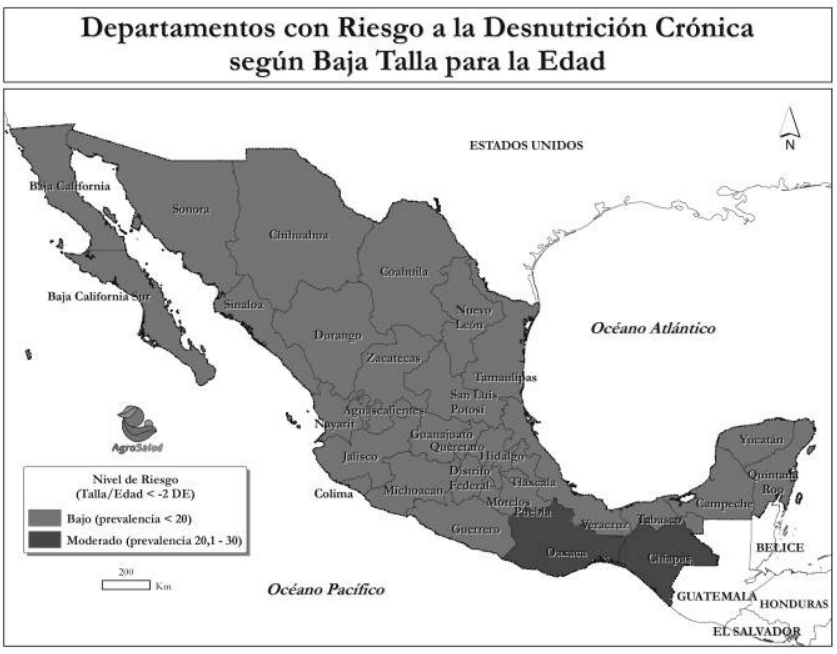

C

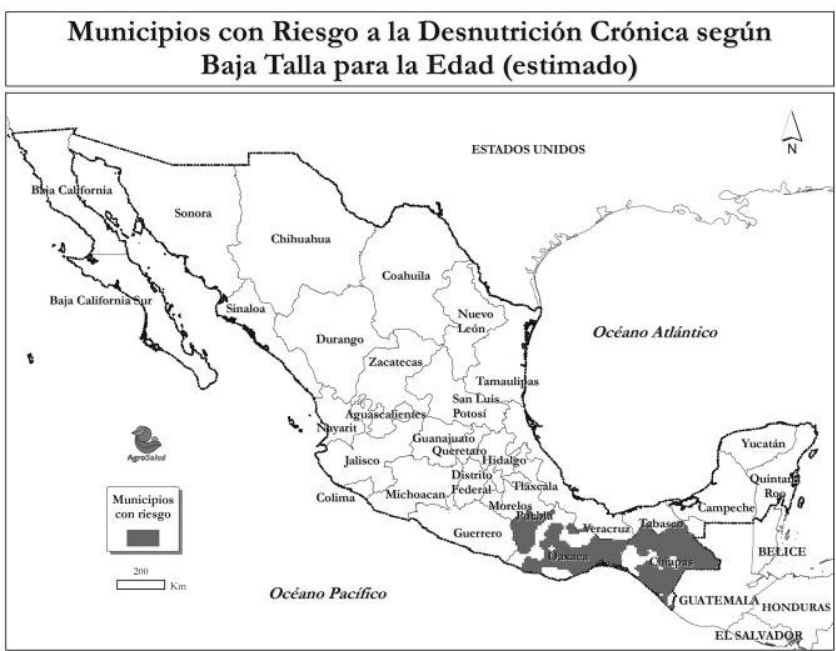

Contra el estándar de oro de mediciones directas a nivel municipal, el esquema conceptual tuvo alta coincidencia $(\geq 66 \%$ ) en las localidades con riesgo a desnutrición crónica. En comparación con un método estadístico indirecto, el esquema no tuve coincidencia alguna $(0 \%)$ en las localidades en riesgo a la desnutrición crónica. Estos resultados sugieren que el esquema tiene buen comportamiento para la identificación de localidades donde hay baja talla/edad, el método EAP se debería evaluar contra un estándar de oro para determinar cuán bien predice municipalidades con desnutrición crónica y el esquema se debería comprobar con estudios de campo en más

B

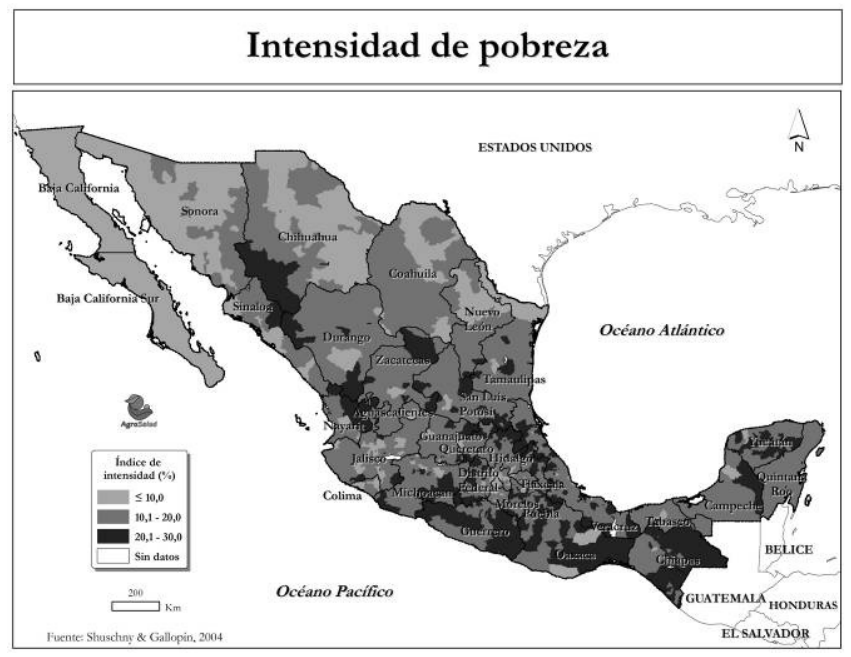

$\mathrm{D}$

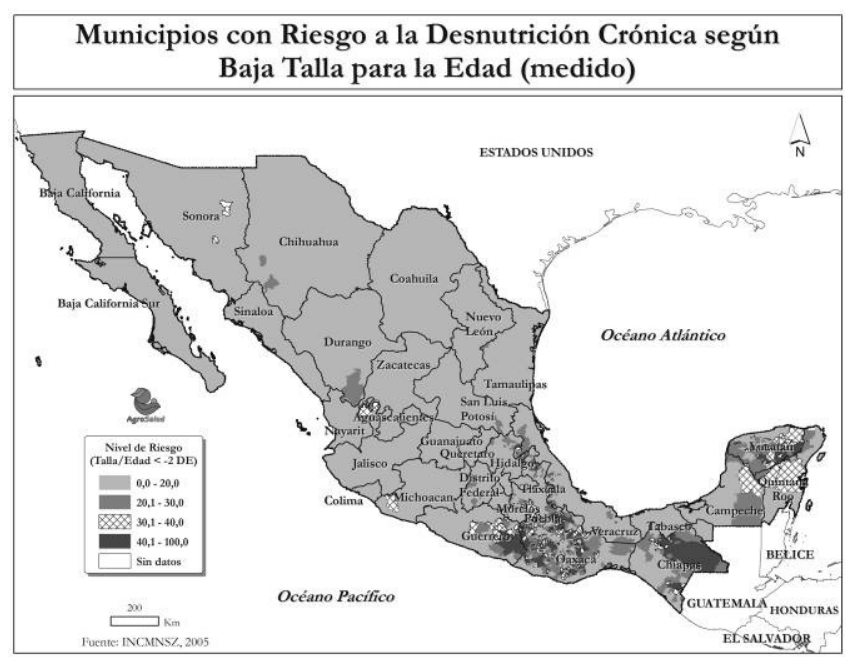

Figura 2. Municipios mexicanos en riesgo a la desnutrición crónica: estimado (mapa C) según un cruce de los Estados con mayor riesgo a baja talla/edad (mapa A), intensidad de pobreza a nivel municipal (mapa B) y medida directamente por investigadores [13] (mapa D). 
Municipios con Riesgo a la Desnutrición Crónica según Baja Talla para la Edad

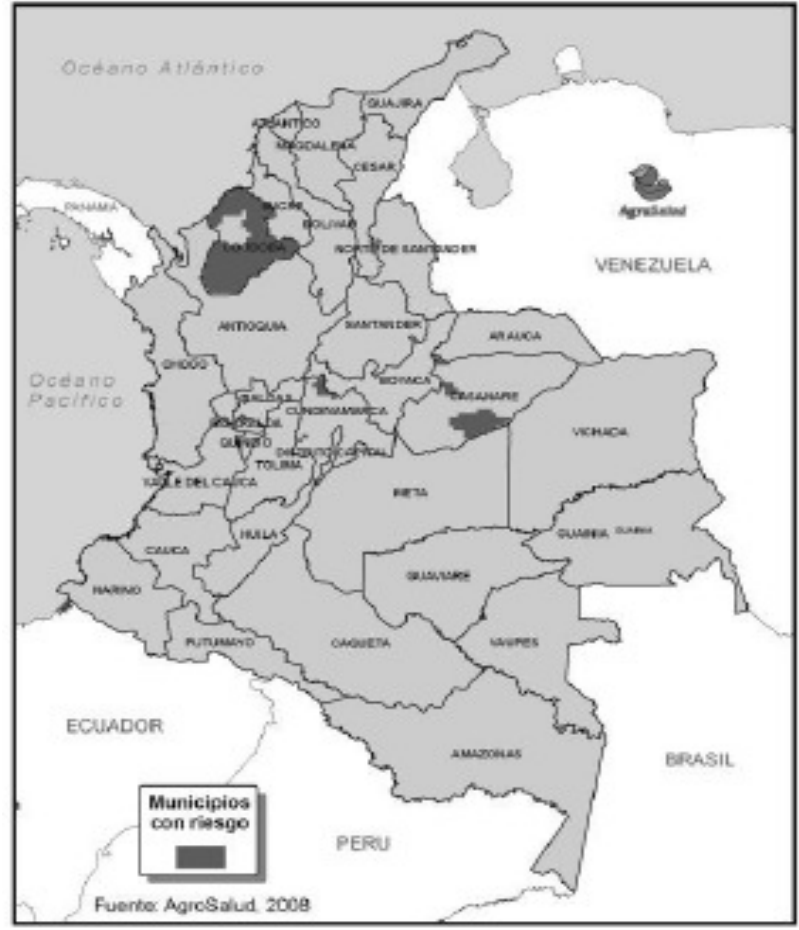

Municipios con Riesgo a la Desnutrición Crónica según Baja Talla para la Edad

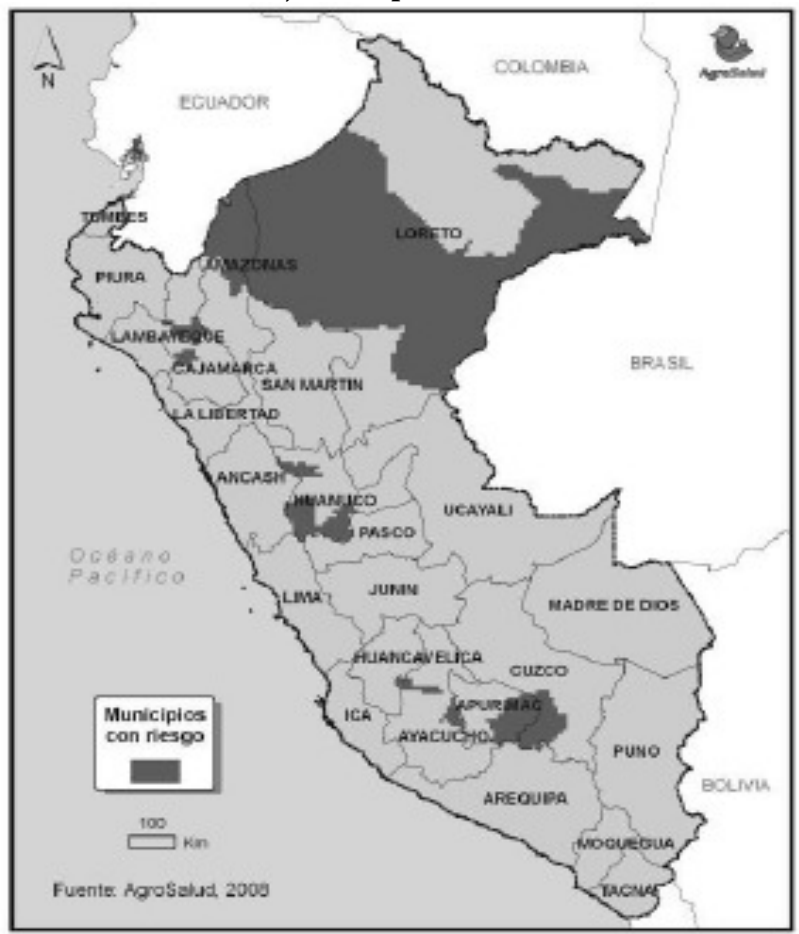

C

Municipios con Riesgo a la Desnutrición Crónica según Baja Talla para la Edad

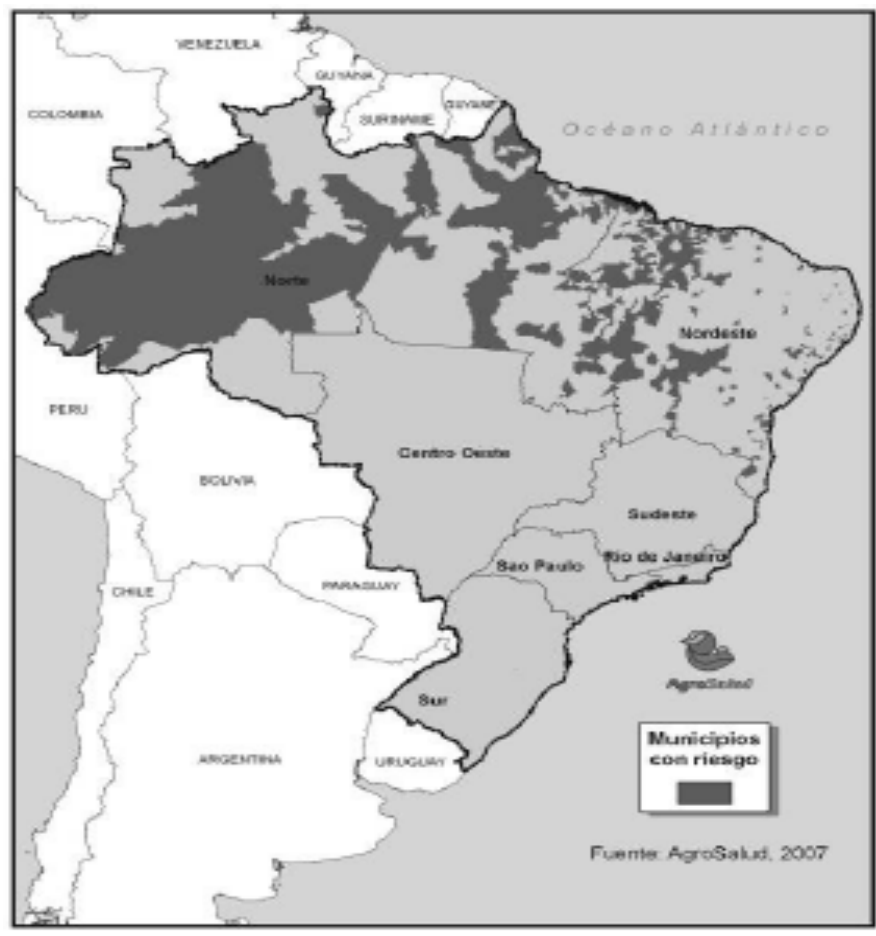


Continuación...

$\mathrm{D}$

\section{Municipios con Riesgo a la Desnutrición Crónica según Baja Talla para la Edad}

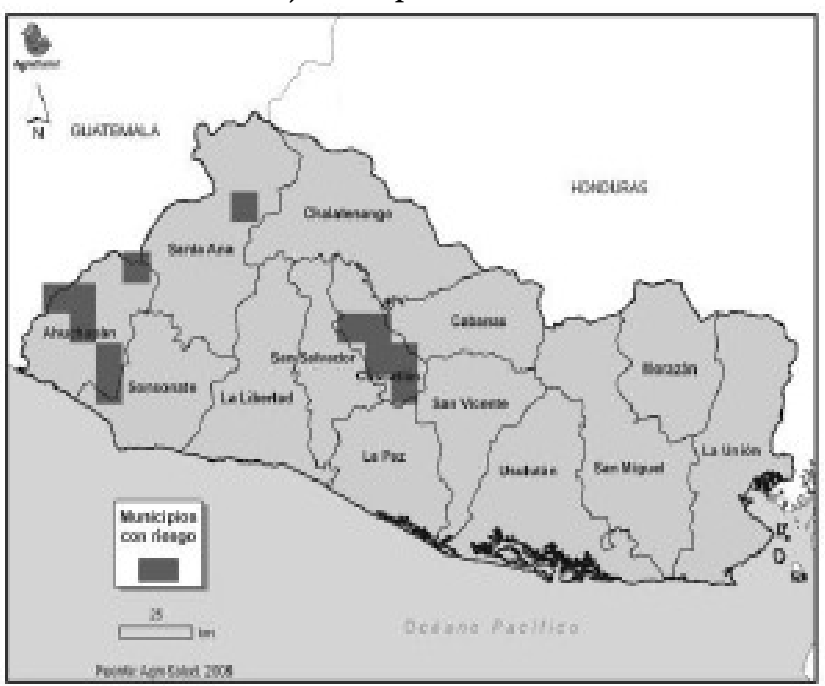

E

\section{Municipios con Riesgo a la Desnutrición Crónica según} Baja Talla para la Edad

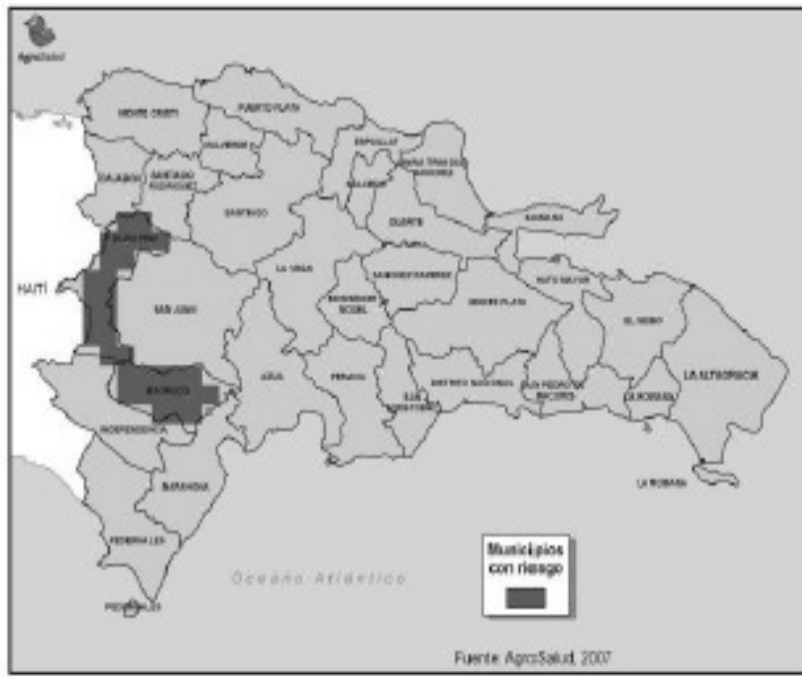

Figura 3. Municipios en riesgo general a desnutrición crónica (Colombia, mapa A; Perú, mapa B; Brasil, mapa C; El Salvador, mapa D; República Dominicana, mapa E).

países, tanto para confirmar los resultados para la desnutrición crónica como para evaluar su comportamiento para predecir localidades en riesgo de anemia.

Durante un diagnóstico para identificar beneficiarios de programas, la FAO [14] recomienda el uso de bases de datos y estudios existentes, versus recolección primaria de datos, como una manera de ahorrar recursos. Esta metodología presentada en el esquema es similar, conceptualmente, a uno de los métodos que presenta la FAO para hacer la focalización de intervenciones: Hacer una evaluación multi-etápica, primero identificando las zonas geográficas donde el problema nutricional es más grave y luego identificando comunidades en riesgo. Además, se puede hacer una focalización a nivel de hogares dentro de comunidades, algo que el esquema conceptual no permite. Según la FAO, cuando la focalización se hace con datos a un nivel geográfico más pequeño (ejemplo: municipio, en vez de departamento), como se pretende con el esquema, mejora la cobertura de programas nutricionales.

Implicaciones para el uso del esquema. En algunos países, seleccionar los lugares donde coincidían mayor desnutrición (según hemoglobina o talla/edad) y mayor pobreza (según datos socioeconómicos), arrojó un número limitado de municipios (e.g., Bolivia, El Salvador) y en otros, un número alto de municipios (e.g., México según hemoglobina) o de una extensión geográfica amplia (e.g., Brasil y Perú según talla/edad). Esto implica, en el caso del segundo, que sólo los mapas no son suficientes para identificar un número limitado de municipios en los que se pueda promover una intervención nutricional y que se deberá tomar en cuenta otros factores relevantes al programa, como accesibilidad y presencia de autoridades gubernamentales.

Al comparar los municipios en riesgo según anemia o desnutrición crónica, se encontró que aparecen más municipios en riesgo, según el primero, debido a que gran parte de los países tienen una mayor prevalencia de baja hemoglobina que de baja talla/edad. Con algunas excepciones, al seleccionar aquellos municipios con riesgo según hemoglobina, se abarcaron simultáneamente los que tienen riesgo según talla/edad, casos presentados especialmente en Colombia y México. En otras palabras, si sólo se cuentan con datos de hemoglobina y no de talla/edad, al usar los primeros se incluirán varios de los mismos municipios identificados con el segundo. 
A

Municipios con Riesgo a la Anemia según Baja Hemoglobina

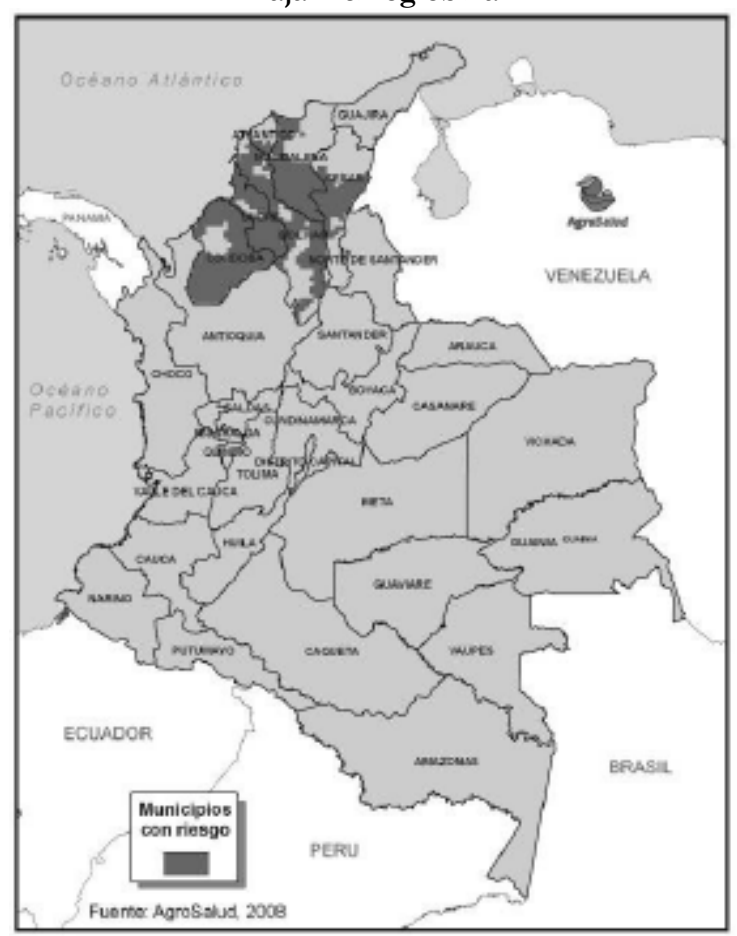

C

Municipios con Riesgo a la Anemia según Baja Hemoglobina

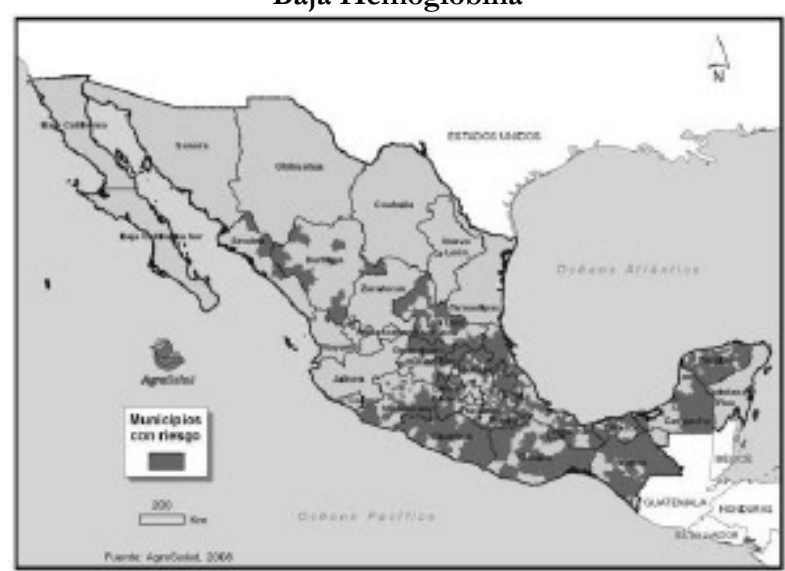

B

Municipios con Riesgo a la Anemia según Baja Hemoglobina

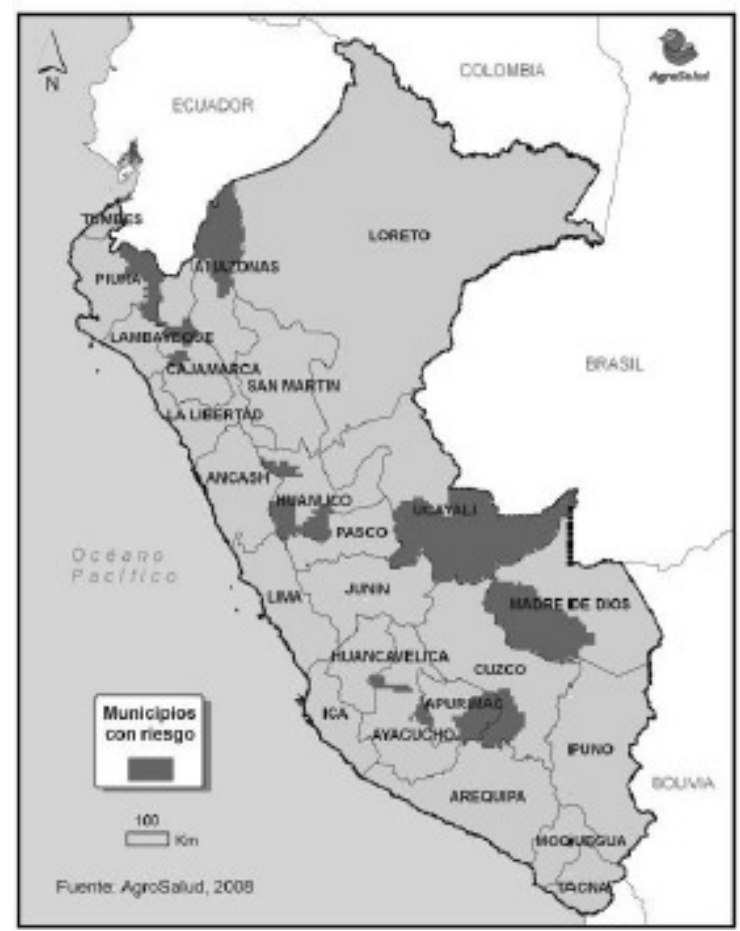

$\mathrm{D}$

Municipios con Riesgo a la Anemia según Baja Hemoglobina

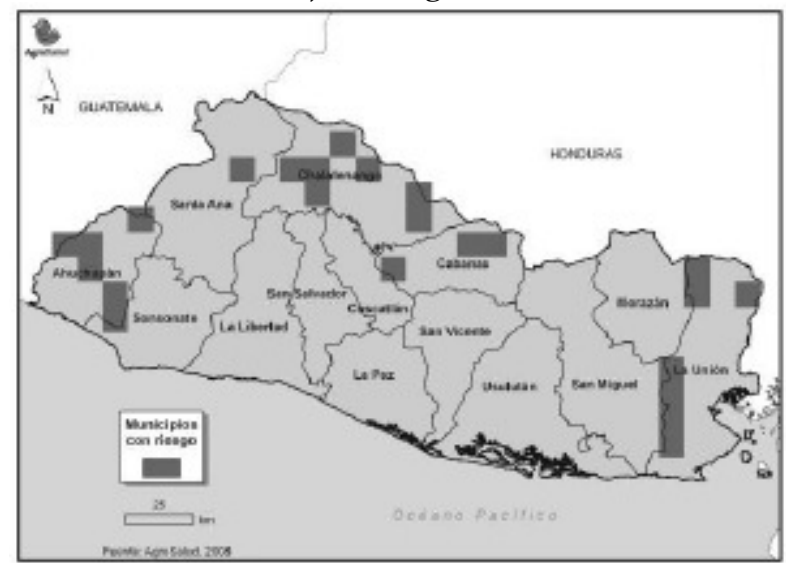

Figura 4. Municipios en riesgo a anemia (Colombia, mapa A; Perú, mapa B; México, mapa C; El Salvador, mapa D).

\section{CONCLUSIONES}

Se desarrolló un esquema que, simultáneamente, considera datos nutricionales y socioeconómicos para identificar localidades donde la desnutrición y la pobreza coinciden, y donde una intervención nutricional debe ser prioritaria. Hubo una alta relación $(\geq 66 \%)$ de localidades identificadas con el esquema, al compararlas con un estándar de oro (datos recolectados a nivel municipal para la desnutrición 
crónica), mas no así ( $0 \%$ ) cuando se comparó con un método estadístico indirecto para identificar localidades con alta probabilidad de tener desnutrición crónica.

Es importante validar el esquema en varios países, tanto para confirmar los resultados encontrados con desnutrición crónica como para evaluar su valor en identificar lugares en riesgo a padecer de anemia. Para esta validación, lo ideal sería que se realizará la recolección en campo de datos antropométricos o bioquímicos, o que se aprovechara bases de datos ya existentes con estos datos, siempre y cuando tengan representatividad municipal. Con estos datos primarios se calcularía la prevalencia de desnutrición e, idealmente, éstos abarcarían municipios con alto, mediano y bajos niveles de desnutrición. Al mismo tiempo, se utilizarían datos existentes a nivel departamental de nutrición y municipal de socioeconomía para aplicar el esquema conceptual y evaluar si coinciden entre sí los municipios con mayor desnutrición. Al confirmar una alta coincidencia, se podría aplicar el esquema conceptual a todos los municipios del país, lo que contribuiría a la identificación de lugares aptos para implementar programas y proyectos de nutrición.

\section{AGRADECIMIENTOS}

Profesora Martha Cecilia Álvarez Uribe de la Universidad de Antioquia, en Colombia, por su participación en la conceptualización del esquema. Dr. Abelardo Ávila Curiel del Instituto Nacional de Ciencias Médicas y Nutrición Salvador Zubirán, en México, por enviar los datos del censo nacional de talla de ese país. Lic. Marlene Rosero del CIAT por su edición del manuscrito. Estudiante en geografía Carlos Ramírez por la búsqueda de bases de datos. El Proyecto AgroSalud (CIDA 7034161) por el apoyo económico para realizar este trabajo y el Programa de Análisis de Políticas del CIAT por su contribución con software, hardware y oficina.

\section{REFERENCIAS}

[1] Lutter CK, Chaparro CM, Muñoz S. Progress towards Millenium Develoment Goal 1 in Latin America and the Caribbean: The importance of the choice of indicator for undernutrition. Bull World Health Organ. 2011;89(1):2230 .

[2] Rivera J, Uauay R. Challenges for childhood health and nutrition research in Latin America, addressing the 90/10 Gap: A regional Latin American agenda for applied health and nutrition research based on current nutritional problems and programs and existing scientific research capacity in the region. Bangladesh: Child Health and Nutrition Research Initiative; 2006.

[3] Organización Mundial de la Salud. Centers for Disease Control and Prevention Atlanta. Worldwide prevalence of anaemia 1993-2005: WHO global database on anaemia. WHO Library; 2008.

[4] Measure DHS. STATcompiler: Building tables with DHS data [citado 2010 febrero 8]. Disponible en: http://www.statcompiler.com

[5] Organización Mundial de la Salud. Preventing and controlling iron deficiency anaemia through primary health care: A guide for health administrators and programme managers. OMS; 1998.

[6] Alive \& Thrive. Insight: Why stunting matters. Washington DC: Alive \& Thrive 2010 [citado 2011 mayo 20]. Disponible en: http://aliveandthrive.org/resource/insight-why-stuntingmatters

[7] Instituto Colombiano de Bienestar Familiar. Encuesta nacional de la situación nutricional 2005. Colombia: ICBF; 2006.

[8] Instituto Nacional de Salud Pública (INSP). Encuesta nacional de salud y nutrición 2006. México: INSP; 2006.

[9] DeMaeyer E, Dallman P, Gurney J, Hallberg L, Sood S, Srikantia SG. Preventing and controlling iron deficiency anaemia through primary health care: A guide for health administrators and programme managers. Geneva: Organización Mundial de la Salud; 1989.

[10] Onis M, Onyango AW, Borghi E, Garza C, Yang H. Comparison of the World Health Organization (WHO) child growth standards and the National Center for Health Statistics/WHO international growth reference: Implications for child health programmes. Public Health Nutr. 2006;9(7):942-47.

[11] United States Agency for International Development. Confronting food insecurity in the LAC region under rising food prices: A framework for action. Washington DC: USAID; 2008.

[12] Instituto Colombiano de Bienestar Familiar. Colombia Nutrida: Seminario Internacional Bogotá Mayo 2010. Bogotá: ICBF; 2010.

[13] Instituto Nacional de Ciencias Médicas y Nutrición Salvador Zubirán. Censo nacional de talla. México: INCMNSZ; 2005. 
[14] Food and Agriculture Organization. Targeting for nutrition improvement: Resources for advancing nutritional well-being. Italia: FAO; 2001.

[15] Muñoz López MdM, Revelo MC, Pachón H. El consumo y la producción familiar de fríjol, maíz, yuca, batata y arroz en un municipio rural en Colombia: Evaluación de la posibilidad de implementar la biofortificación de cultivos. Persp Nutr Humana. 2008;10(1):11-21.

[16] Pachón H. El impacto nutricional de cultivos biofortificados o cultivos con mayor calidad nutricional. Colombia: Centro Internacional de Agricultura Tropical (CIAT); 2010.

[17] Denova-Gutiérrez E, García-Guerra A, FloresAldana M, Rodríguez-Ramírez S, Hotz C. Simulation model of the impact of biofortification on the absorption of adequate amounts of zinc and iron among Mexican women and preschool children. Food Nutr Bull. 2008;29(3):203-212.

[18] Gómez González LK, Restrepo J, Pachón H. Caracterización del consumo de maíz y fríjol en familias del departamento del Cauca, Colombia. Persp Nutr Humana. 2010;12:87-98.

[19] Arsenault JE, Yakes EA, Hossain MB, Munirul Islam M, Ahmed T,Hotz C, Lewis B, Shafiqur Rahman A, Jamil KM. The current high prevalence of dietary zinc inadequacy among children and women in rural Bangladesh could be substantially ameliorated by zinc biofortification of rice. J Nutr. 2010;140(9):1683-90.

[20] Haas JD, Beard JL, Murray-Kolb LE, del Mundo AM, Felix A, Gregorio GB. Iron-biofortified rice improves the iron stores of nonanemic Filipino women. J Nutr. 2005;135(12):2823-30.

[21] Haas JD, Villalpando S, Beebe S, Glahn R, Shamah T, Boy E. The effect of consuming biofortified beans on the iron status of Mexican school children. FASEB J. 2011 [citado 2011 junio 03]. Disponible en: http://www.fasebj.org/cgi/content/meeting abstract/25 $\angle 1$ MeetingAbstracts $/ 96.6$ ? sid=183b09b2-235e-4ea2a1cb-c2e59c7d35be

[22] Rosado JL, Hambidge KM, Miller LV, Garcia OP, Westcott J, Gonzalez K, Conde J, Hotz C,Pfeiffer W, Ortiz-Monasterio I, Krebs NF. The quantity of zinc absorbed from wheat in adult women is enhanced by biofortification. J Nutr. 2009;139(10):1920-25.

[23] Gunaratna NS, De Groote H, Nestel P, Pixley KV, McCabe GP. A meta-analysis of community based studies on quality protein maize. Food Policy. 2010;35(3):202-10.
[24] Organización Mundial de la Salud. A growth chart for international use in maternal and child health care: Guidelines for primary health care personnel. Geneva: OMS; 1978.

[25] Organización Mundial de la Salud. The WHO child growth standards. Ginebra: OMS; 2006.

[26] Centro de Estudios Sociales y Demográficos. Encuesta demográfica y de salud 2002. República Dominicana: CESDEM; 2002.

[27] Shuschny A, Gallopín G. La distribución espacial de la pobreza en relación a los sistemas ambientales en América Latina: Proyecto "Evaluación de la Sostenibilidad en América Latina y el Caribe". Chile: Naciones UnidasCEPAL; 2004.

[28] Feres JC, Mancero X. El método de las Necesidades Básicas Insatisfechas (NBI) y sus aplicaciones en América Latina. Chile: Naciones Unidas-CEPAL; 2001.

[29] Wood S, Guo Z, Koo J, Hyman G, Herrero M, Tenorio R, Williams O, Wood-Sichraa U, Sebastian K. Geographic domain analysis to support the targeting, prioritization and design of a CGIAR mega-project (MP) portfolio. USA: CGIAR Alliance 2009 [citado 2010 marzo 5]. Disponible en: http://alliance.cgxchange.org/documentation-for-thedevelopment-of-the-cgiar-strategy-and-mega-programs

[30] Food and Agriculture Organization. Indicadores de nutrición para el desarrollo. Italia: FAO; 2006.

[31] Organización Mundial de la Salud. Iron deficiency anaemia: Assessment, prevention, and control, a guide for programme managers. OMS; 2001.

[32] Zapata-Caldas E, Hyman G, Pachón H, Monserrate $\mathrm{F}$, Vesga L. Identifying candidate sites for crop biofortification in Latin America. Int $\mathrm{J}$ Health Geogr. 2009;19:8:29.

[33] Rogers BL, Macías KE, Wilde P. Atlas del hambre y la desnutrición en la República Dominicana. República Dominicana: Programa Mundial de Alimentos; 2007.

[34] Lepkowski JM. Sampling the difficult-to-sample. J Nutr. 1991;121(3):416-23.

[35] Instituto Nacional de Estadística Boliviano. Encuesta nacional de demografía y salud. Bolívia: INE; 2003.

[36] Asociación Demográfica Salvadoreña. Encuesta nacional de salud familiar. El Salvador: ADS; 2003.

[37] Instituto Nacional de Estadística. Encuesta nacional de salud materno infantil 2002. Guatemala: INE; 2002. 
[38] Institut Haïtien de l'Enfance. Enquête mortalité, morbidité et utilisation des services. Haití: IHE; 2001.

[39] Secretaría de Salud, Instituto Nacional de Estadística y Macro International. Encuesta nacional de salud y demografía 2005-2006. Honduras: SS, INE y Macro International; 2006.

[40] Instituto Nacional de Estadísticas y Censos. Encuesta nicaragüense de demografía y salud 2001. Nicaragua: INEC; 2001.
[41] Instituto Nacional de Estadística e Informática. Encuesta demográfica y de salud familiar 2000. Perú: INEI; 2001.

[42] Sociedade Civil Bem-Estar Familiar no Brasil. Pesquisa nacional sobre demografia e saúde 1996. Brasil: BEMFAM; 1997. 